\title{
VARIA
}

\section{LA QUINTA DEL SORDO EN 1830: RESPUESTA A NIGEL GLENDINNING.}

Según el Diccionario de la Real Academia de la Lengua Española precisión es un vocablo que se define de la siguiente manera: 1. «Obligación o necesidad indispensable que fuerza y precisa a ejecutar una cosa». 2. «Determinación, exactitud, puntualidad, concisión».

A la vista del artículo de Nigel Glendinning Las Pinturas Negras de Goya y la Quinta del Sordo. Precisiones sobre las teorías de Juan José Junquera ${ }^{1}$, parece que él se acoge a la primera acepción; yo lo haré a la segunda, por lo que no insistiré en mis «teorías» ya expuestas en Conocer el Arte, mi libro The Black Paintings of Goya (Londres, Scala, 2003) y en A.E.A. ${ }^{2}$ sino que me limitaré a algunas precisiones acerca de las suyas, aunque me extraña que no se cuente entre los «lectores avisados» a los que invoca puesto que, en las páginas 33 y 34 - tanto de la edición inglesa como de la española del libro- queda claro cómo era la casa en tiempos de Francisco de Goya pero, para despejar las dudas, reproduzco el inventario de la Quinta redactado en diciembre de 1830, cuando pasa a poder de su nieto Mariano, el futuro falso Marqués del Espinar ${ }^{3}$. En el documento - que constituye la más antigua descripción de la casa y sus chismes hoy conocida - se refleja con una minuciosidad extraordinaria todo lo que contenía la posesión, desde los animales de labor hasta el abono que se estaba formando en el pudridero, pasando por los comestibles que guardaba la despensa y sin olvidar los muebles. Estos aparecen con la denominación utilizada en vida de Goya; así nos encontramos una papelera y no un bargueño, un mueble de charol, que no de maque,y lo más interesante de todo ocho cuadritos de varios asuntos pintados por su abuelo, y cedidos por el, identificación que — como su precio, el de unos bocetos ${ }^{4}$ — no se acomoda con las Pinturas Negras, de las cua-

\footnotetext{
1 Nigel Glendinning: «Las pinturas Negras de Goya y la Quinta del Sordo. Precisiones sobre las teorías de Juan José Junquera», A.E.A. LXXVII, 2004, 307, pp. 233 a 245.

2 Juan José Junquera y Mato: «Las Pinturas Negras, bajo sospecha», Descubrir el Arte, año V, n 51, mayo 2003 , pp. 23-32. Juan José Junquera: «Los Goya: de la Quinta a Burdeos y vuelta», A.E.A, LXXVI, 2003, pp. 353 a 370.

${ }^{3}$ Archivo histórico de Protocolos de Madrid. Legajo $n^{\circ}$ 22887. Antonio López de Salazar.

Capital de los bienes que lleva/ a su matrimonio D. Pio Mariano/ de Goya, otorgado por su futura Es/posa Da Maria Valvanera de la Concep/ cion Mariategui, y por sus Padres D. Franco/ Xavier de Mariategui, y Da Ramona Asperilla. [EI documento se reproduce al final del texto del artículo].

${ }^{4}$ Sobre el precio de los bocetos de Goya, véase: N. Glendinning: «Spanish inventory references to paintings by Goya,1800-1850: originals, copies and valuations», The Burligton Magazine, vol. CXXXVI, $\mathrm{n}^{\circ}$ 1092, marzo 1994, pp. 100110. José Manuel de la Mano: «La idea sobre el lienzo: Gestación, función y destino del boceto en Goya y sus contemporáneos», en Goya y Maella en Valencia. Del Boceto al Cuadro de Altar, Valencia, marzo-mayo 2002, pp. 42-57. Los dos bocetos de San Antonio de la Florida de la antigua colección Villagonzalo, subastados en Madrid - Alcalá Subastas, Madrid, octubre 2004, n $\mathrm{n}^{\circ} 72$ - estaban tasados en la colección de su poseedor coetáneo de Goya D. Silvestre Collar en 300 reales, mientras que otro, de la misma colección — también de Goya - una «Anunciación de N ${ }^{a}$ Señora, se evaluaba en 200
} 
les no hay rastro, hecho especialmente notable cuando, para todo lo demás, el inventario es absolutamente explícito. Debemos resaltar también cómo, al igual que en el reparto de bienes entre Goya y su hijo de octubre de 1812, no aparece ningún libro, lo cual nos debe llevar a replantearnos el supuesto carácter «ilustrado» de Goya que su correspondencia desmiente.

Acompaño unas plantas - tomadas de Kentish-Glendinning, con lo que creo se desprende de éste documento y del de la compra de la Quinta por Goya- que son las probables de la primitiva casa y de la ampliación o «aumento» hecha por Goya en el piso bajo, único entonces existente. En cualquier caso le recomiendo a Nigel Glendinning la lectura de mi trabajo Cortés, los Colón y la «villa» en el Mundo Hispánico ${ }^{5}$, que le ayudará a comprender el porqué de lo magro de nuestras casas de campo y el poco aprecio que, los españoles, hemos tenido a la vida rural desde la época de los Trastámara y hasta la llegada del chalet adosado. También le sería de interés mi libro La Decoración y el Mobiliario de los Palacios de Carlos IV, donde se estudia la arquitectura de las casas de campo del rey así como su relación con el paisaje, sin olvidar la Real Fábrica de Papel Pintado de D. Pedro Giroud de Villette, de la cual se reproduce una factura que ilustra acerca de su producción que nada tiene que ver con los de la Quinta, de la época de Mariano.

En cuanto a las fotos de Laurent, se debieron tomar en la Quinta pero posiblemente en el momento en el que se iba a proceder al arranque de las «Pinturas Negras», y no ya en el $\mathrm{Mu}$ seo del Prado como yo había sugerido; lo cual parece quedar confirmado por lo visto en la exposición El Grafoscopio. Un siglo de miradas al Museo del Prado 1819-1920 ${ }^{6}$.

Esto, como todo lo demás contenido en las Precisiones de N. Glendinning se refiere, siempre, a época tardía, muchos años después de la marcha definitiva de Goya (Francisco) a Burdeos.

Me ha resultado sorprendente su aguda observación en la que relaciona la expresión «estilo Luis XV» que aparece en el «Inventario Brugada» con la obra de Monsieur de Voltaire Le Siècle de Louis XIV. Desgraciadamente, la Inquisición y los sucesivos Ministerios ya se ocuparon con harta eficacia en que los mohatreros y vendedores de antigüedades del entorno de la josefina Plaza de Santa Ana no pudieran leer a François Marie Arouet. Tuvieron que esperar hasta la muerte de Fernando VII para permitirse tales licencias bibliográficas y semánticas. Una pena.

Mesonero Romanos, hombre práctico, en su ancianidad y junto a una estufa «a la prusiana» que no atufaba como el viejo braséro, quizás pensara por qué Goya (Javier) había pintado al óleo y en las paredes de una habitación donde su padre, quizás, lo había hecho al fresco, como en la escalera que subía al zaquizamí, y cuál fue el motivo que le llevó a continuar haciéndolo en el piso superior recién construído de una casa en cuya verja de entrada, Marianito había colocado unas iniciales timbradas por una corona que no le correspondían, lo cual ya desde Felipe II constituía un «crime» aunque nosotros, más circunspectos con el término, sólo lo tildemos de «delito».

reales. V: Juan José Junquera: Goya en San Antonio de la Florida: La idea, los bocetos y un coleccionista, de inminente publicación.

5 Juan José Junquera:«Cortés, los Colón y la villa en el Mundo Hispánico», A.E.A, 242, 1.988, pp.95-104. Id.: La Decoración y el Mobiliario de los Palacios de Carlos IV, Madrid, 1979.

${ }^{6}$ El Grafoscopio. Un siglo de miradas al Museo del Prado (1819-1920), 22 de junio- 26 de septiembre 2004.

AEA, LXXVIII, 2005, 309, pp. 83 a 105 


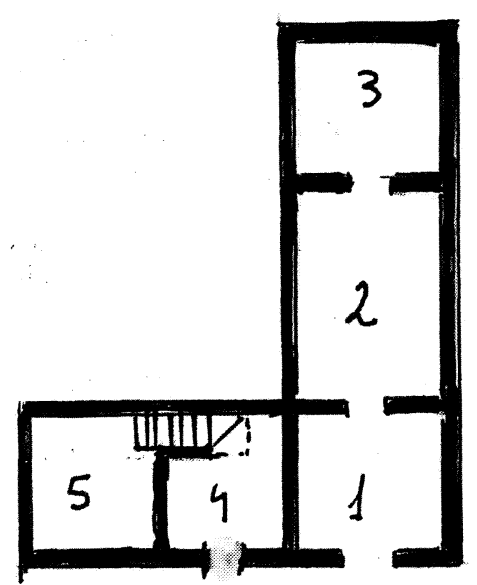

Fig 1. La Quinta del Sordo en 1819:

$\begin{array}{ll}\text { Habitación principal: } \\ \text { 1. } & \text { Antesala. } \\ \text { 2. } & \text { Sala. } \\ \text { 3. } & \text { Gabinete. }\end{array}$

Habitación del hortelano:

4. Cocina.

5. Cuarto.

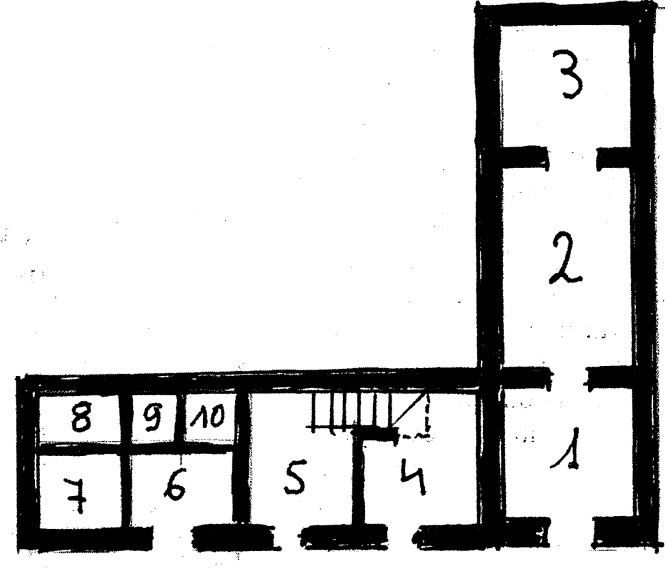

Fig. 2. La Quinta del Sordo en 1830 :

Habitación antigua:

$1 .^{1 / 4} \quad$ Antesalá

-.... Sala

3. Gabinete

4 y. 5 - Antigua habitaciôn del hortelano.

$6,7,8,9$ y 10 cuartos añadidos por Francisco de Goya.

\section{APÉNDICE DOCUMENTAL}

\section{[Inventario de la Quinta redactado en diciembre de 1830]}

«Archivo histórico de Protocolos de Madrid. Legajo n 22887. Antonio López de Salazar.

Capital de los bienes que lleva/ a su matrimonio D. Pio Mariano/ de Goya, otorgado por su futura Es/ posa $\mathrm{D}^{\mathrm{a}}$ Maria Valvanera de la Concep/ cion Mariategui, y por sus Padres D. Franco/ Xavier de Mariategui, y Damona Asperilla.

\section{En 9 de/ Diciembre de 1830}

(Al margen: En dicho dia di/ dos copias en pa-/ pel de sello de/ suf piimer \% pliego e interrmedio del cuarto/ mayor una pa/D. Franco. Xavier/ de Goya y Bayeu/ y otra para D. Pio/ Mariano de Goya / y Goicoechea/ doy fe

En la Villa de Madrid a nueve/ de Diciembre de mil ochocientos treinta. Ante mi/ el Escribano de S. M. De Camara del Real y Supremo Consejo de Castilla, y testigos parecieron D ${ }^{a}$ Maria Valvanera de la Concepcion de Mariategui y Aspe/ rilla de estado Soltera de edad de veinte y tres años/ natural de esta Corte, D. Francisco Xavier de Mariategui, y D ${ }^{a}$ Maria Ramona Asperilla/ aquel Teniente de Arquitectó mayor de esta villa/ de donde son vecinos. Dixeron: Que a honra/ y gloria de Dios N. S. y para su Santo Servi-/cio esta tratada la primera de casarse in facie/ Eclesia con D. Pio Mariano de Goya y Goicoechea/ tambien de estado Soltero de edad de veite y cutro/ años, y natural de esta misma Corte, hijo lejiti/ mo de D. Francisco Xavier de Goya y Bayeu y / (fol 26) /

de $\mathrm{D}^{\mathrm{a}}$ Maria Gumersinda de Goicoechea y Ga/larza ambos naturales y vecinos de esta misma villa/ a cuyo fin han precedido las amonestaciones que/ previene el Santo Concilio de Trento, y respecto a/ que los citados D. Francisco Xavier Pedro de Goya/ y Da Maria Gumersinda de Goicoechea, y Ga/ larza han prometido a su hijo D. Pio darle/ en diferentes bienes efectos, y dinero la cantidad/ de Setenta y ocho mil ochocientos ochenta y dos/ Rs.Von por cuenta de su lejitima paterna y/ materna, y ademas lleva doscientos Seis mil qui/ nientos veinte y cinco Rs. De que le hizo dona.on/ su Abuelo D. Francisco Goya, Pintor de Cama/ ra que fue de S. M. Cuyas partidas as/ cienden a la Suma de doscientos ochenta y cinco/ mil cuatrocientos Siete Reales para atender/ a las cargas del matrimonio, vajo la calidad/ de que se formalice a su favor la correspondien/ te Escriyura de Capital, poniendolo en exe/ cucion por la presente: OTORGAN: Que/ el citado D. Pio Mariano de Goya y Goicoe/chea trae por capital suyo los bienes sigtes/

(al margen: «Bienes do/ nados por su/ Abuelo D Franco. De Goya»)

Primeramente una posesion extra/ muros de esta Corte pasado el Puente/ de Segovia, que linda con el Camino/ (fol26 vto.) 
(al margen derecho: "Rs von») que desde este dirige a la hermita de / san Isidro, y se compone de catorce fa/ negas de tierra, seis de regadio, y ocho/ de secano con una casa de recreo, $\mathrm{Ca}$ / sa de Labor, cuadras, noria, estanque, y/ por una parte un trozo de cercado de / Pared, que le fue cedido graciosamente/ el diez y siete de setiembre de mil ocho/ cientos veinte y tres por su Abuelo D Frco,/ de Goya y Lucientes valuada en cien-/ to noventa y nueve mil veinte y cinco Rs 199.025/ Itm. Una Joya de brillantes cuadrada/ en forrma de collar con cuatro cadenas/ de oro regalada por dicho su Abuelo/ apreciada en Cuatro mil RsVon......4.000/ Itm. Ocho Cuadritos de varios asuntos pintados por su Abuelo, y cedidos por el/ en dos mil quinientos Rs.......2.500/ Una mesa de Caova Inglesa de la que se/ forma cuatro Atriles para musica re/ galada al D. Pio por su tio D. Mariano de Goicoechea y Galarza en mil Rs....!000/ 206.525 (fol 27)

(al margen: «Bienes/ dados por sus padres) (al margen derecho: 206525) En Mejoras hechas en la Posesion/ Un Estanque para labar Verdura; un Coberti/ zo para poner los carros, y en la noria dos pi/lares con su viga jabalconada valuan/do todo en Siete mil quinientos Rson....7.500/ Itm. Un Jardin que esta en la casa / ciento treinta y nueve frutales, una/ injertera, y mil trscientos cinquenta/ y seis varas de cerca de cambroneras, y/ almendros valuado en nueve mil qui/ nientos veinte y dos Rs.....9.522/ Itm. Cuatro Mulas en tres mil seztos rs....3700/ Utm. Un Carro un poco usado en mil/ doscientos Rs.......1200/ Itm. . otro mas usado en setecientos, y/ Setenta.....770/ Itm. Todas las guarniciones cabezadas/ en setecientos Rs...700/ Itm. Una Borrica en cuatrocientos Rs....400/ Itm. Alvarda Serones, aguaderas pa-/ ra la dicha en Sesenta.....60/ Itm. Un Arado completo en cuarenta/ y Seis....46/ Itm. Tres rejas de Arado en cien Rs....100 / Itm. Una tapilla en trescientos Rs.on........300/ 230.829 (fol 27vto).

Itm. Tres carretillas en ciento cuarenta....140/ Item. Tres Azadones de Gancho en ochenta......80/ Itm. Seis Azadones de pala nuevos doscien/tos cuarenta......240. / Itm. Cuatro Id. Un poco usados Ciento....!00/ Itm. Cinco Id. Muy inferiores treinta...100/ Itm. Seis piquetas ochenta......80/ itm Cuatro palas de yerro y madera/ en diez y seis.....16/ Itm. Cuatrocientos noventa y Seis pares/ de cuerda para maroma doscientos/ sesenta y dos......262./ Itm. Cincuenta docenas de soguilla para/ dicha ciento seis.......106./ Itm. Torno para hacer la Maroma dos/ cientos cinquenta.....250./ Itm. Setecientos cincuenta y un Capazos/ para calabazas doscientos......200/ itm. Una escopeta y un fusil cien/to treinta.......130./ La Basura existente en el Pudride/ro y misma Basura extendida en/ toda la huerta Cuatro mil Rs.....400000/ 236.457.( fol 28)

Itm. Cien eras plantadas de yerba buena/ Seiscientos Rs.......600/ Itm. dos mil y cuatrocientas eras plan/ tadas de lechuga cevolla mil dosz.tos......1200/ Itm. Veinte y cinco eras de Alcacho/ fas ciento....100/ media fanega de Simiente de Escaro/ la en ciento veinte......120/ Item media id de Espinaca noventa y seys......96/ Itm. tres libras de Simiente de pimientos/ comun ciento ochenta....180/ Itm. una id de pimientos de orihuela/ en ochenta reales........80./

(al margen izquierdo: Alajas)

Una repeticion de oro en mil dosctos.. ......1200/ Itm.os cadenas de relox de oro en mil ....1000/ Itm. una Docena de cubiertos de plata a / marillo mil Seiscientos ocho........1608/ Itm. un Cucharon Sopero y otro de pala/ de Id en cuatrocientos uno......401/ Itm. doce Cuchillos con mango de Marfil/ ciento treinta y dos Rs........132/ Itm. Seis Id con mango de Ballena/ treinta y seis........36/ itm. un braserillo de fumar de plata ciento sesenta.....160/ Itm. Dos porta botellas veinte......20/ 2433.390 (fol. 280 vto.).

Itm. un relox de Sobremesa en mil y / doscientos........1200 / Itm. una Lampara de bronce con cuatro quin/ ques mil cien rs....1100/ Itm. dos candelabros de Id. Doscientos..........200./ Itm. un belon de Id ciento cuarenta.........140./ Itm. dos floreros doscientos Sesenta.....260/ Itm. Seis Rinconeras de caova y marmol/ doscientos cuarenta rs........240/ Itm. dos Sillerías para Sala y Gavinete/ mil Seiscientos cincuenta y ocho........16588/ Item. Doce Sillas con asientos verdes doscientos veinte y ocho........228/ Itm.. doce Sillas de Vitoria ciento ochenta.......180./ Itm. una pantalla de chimenea de cao/ va treinta...........30/ Itm. Morillos, tenaza fuelles, para/ chimenea doscientos.....200/ itm. una Escopeta de dos cañones Seiztos.......600/ Itm. una Papelera de Caoba cuatroz.tos......400/ Itm. Un Tocador Papelera quinientos.......500/ Itm un Catre de caova de dos cavecel 250.326 (fol. 29).

ras de caova Seiscientos.........600 / Itm. Cuatro Colchones de cuti sencillo/ para dicho Cuatrocientos........400/ Itm. Colgadura de percal con flores para/ dicho ciento veinte.........120 / Itm.. dos fundas de dicho veinte......20 / Itm. dos rinconeras pintadas treinta.....30/ Itm. un Belador de caova ciento.....100/ Item un Brasero completo ciento ochenta.....180 / Itm. una mesa de comer noventa.....90/ Itm.Esterado de la casa Seiscientos quarta........640/ Item una bandeja de charol catorce......14/ Itm. Llamadores y cintas Setenta......70/ Itm. dos Labamanos diez y Seis......16/ Itm. un piano de cola once mil quin.tos.....11.500 / Itm. un biolin su autor ortega dosmil.....2.000/ Itm. otro de Guadañini en mil......1000/ Itm. otro de Guarnerios Seiscientos.....600/ Itm. dos violas cuatrocientos....400/ Itm. un nArco de violin con caja cuatroz..tos......400/ Itm. un metronomo Ciento Sesenta.....160/ Itm. Itm. Dos atriles cuatrocientos........400/ Itm. una Colección de Estudios para vio/ lin quinientos........500/ Itm. Veinte y dos composiciones para id/ de diferentes Autores, quinientos.....500/ 270.066 (fol 29 vto).

Itm. nueve Conciertos para Id de Yssoti/ de doscientos sesenta.........260/ Itm. once Id de Kreutzer en doscientos/ Sesenta y ocho.....268/ Itm. veinte y tres composiciones para Id/ de Lafont Seiscientos........600/ itm. Veinte Composiciones de Rode qui/ nientos ochenta.......580./ Itm. diez y siete Id de Baillot

AEA, LXXVIII, 2005, 309, pp. 83 a 105 
cuatroz.tos......4000/ Itm. una Colección de trios de Seis Au/ tores, doscientos.......200/ Itm. otra Id de Cuartetos de Cinco Auto/ res doscientos treinta.......230/ Itm. un tratado de Armonia por Ca-/ tel cinquenta........50./.Itm. una Colección completa de cuartetos de Hayden (sic) nueva ediccion en pa-/ pel vitela seiscientos.......600/ Itm. Id de las obras escogidas de Mozart/ doscientos Sesenta........260./ Itm. Id de Id de las de Kromer trescientos/ cuarenta........340/ Itm. Id de Id de las de Beethoven dosc.tos....2000/ Itm. un Juego de Manteleria del / 274.054/ (fol 30).

Bearne con raya encarnada de doce Ser/ villetas, un mantel grande, y un man/telito doscientos ochenta

280./ Itm. dos manteles finos de sesenta y dos/ y medio ciento veinte y cinco....125./ Itm. ocho servilletas de raya azul a / diez y Seis Ciento veite y ocho.......128./ Itm. tres manteles de fam.a cuar.ta y ocho.......48./ Itm. tres Servilletas ordinarias de gusani/ llo doce......12./ Itm. cuatro tohallas del Bearne con raya/ encarnada a diez y seis sesenta y cuatro.......64./ Itm. cuatro Id para fam.a veinte y qtro. ........24/ Itm. dos Savanas de costura para catre/ y cuatro almoadas de Id guarnecidas de / muselina doscientos Sesenta y dos........262./ Itm. Una manta treinta y tres.....33/

Itm. dos Savanas de catre para debajo/ ciento ocho Rs......108/ Itm. ocho savanas de familia doscientos ochenta y cuatro........284/

Itm. ocho almoadas de fam.a cinqta. Y seis.......56/ Itm. dos sobrecamas de fam.a Set ta. y dos......72/ Itm.. catorce cortinillas de muselina p.a/ vidrieras, doscientos diez............21000./ Itm. dos Savanillas p ${ }^{\mathbf{a}}$, planchar v.te y cuatro.......24./ 275.784(fol 30 vto.)

Itm. tres talegos chicos diez......10/ Itm. dos Id grandes catorce.......14/ Itm. tres delantales de cocina quince.........15/ Itm. doce rodillas treinta........30/ Itm. cinco colchones de fam.a . tresc.tos........300/ Itm. una Escribania cinquenta........50./ Itm. una compotera de cristal ocho.........8/ Itm. Unas vinagreras con portavinagre/ras en veinte Rs......20/ Itm. dos Botellas de cristal treintay dos.......32/ Itm. una docena de vasos de cristal veinte y cuatro............24./ Itm. una Sopera de la Moncloa treinta.........30/ Itm. dos jarras de Id treintay seis.....36/ Itm. dos jofainas de Yd veinte....20/ Itm. dos orinales de Id veinte y dos........22/ Itm. cinco docenas de platos de la Moncloa/ ciento y dos Rs........102/ Itm. dos fuentes grandes treinta y dos.........32,/Itm. cuatro fuentes largas cinqta. y seis ........56/ Itm. cuatro Id cuadradas treinta y dos.......32./ 276617. (fol 31).

Itm. dos Id chicas ocho.....8/ Itm. id Cafeteras veinte y dos.....22/ Itm. dos Tazas grandes dos......2/ Itm. un Azucarero cinco.....5/ Itm. un mantequero cuatro.....4/ Itm. seis platos de postre siete.......7/ Itm. una Ensaladera diez.......10/ Itm. Seis jicaras seis.....6/ Itm. Seis tazas y platillos doce......12/ Itm. tres planchas sesenta.....60/ Itm. un fregadero con dos Artesones cin/ quenta y cuatro.....54/ Itm. una mesa de cocina cincuenta y seis......56./ Itm. una Artesa con su pie y tabla de Jabonar noventa......90/ Itm. un tajo treinta......30/ Itm. un cubo y cogedor veintte y siete......27/ Itm. un Cucharero Veinte y Seis.....26/ Itm. Tinaja con pie y tapa veinte y dos......22/ Itm. un jarro de cobre cuarenta.....40/ Itm, un Almirez treinta y cuatro.....34/ Itm. tres sartenes diez y nueve.......19/ Itm. un Perol Sesenta.......60/ Itm. dos Belones de familia treinta y dos......32/ Itm. otro Id de pantalla diez y seis......16/ Itm. tenazas y cogedor de yerro ocho......8/ Itm. un brasero de Yerro con tarima./ 277267 (fol. 31 vto.)

Y badilla treinta y ocho Rs......38/ Itm. Vidriado ordinario Sesenta.....60/ Itm. Espumadera y cacillos seis......6/ Itm. trevedes, y dos coberteros veite y seis......26/ Itm. unos fuelles diez.....10/ Itm. Cuchillo y cuchilla de Cocina quince......15/ Itm. Aceitera, Cafetera, embudo y rallo de oja/ de lata veinte y seis.....26/ Itm. dos Cepillos veinte.....20/ Itm. ocho a(rrobas) de Garbanzos dosc.tos. sesenta y cuatro....264/ Itm. Tres tareas de chocolate mil set.ta. y cinco.....1075./ Itm. dos a(rrobas) de Jabon noventa y cuatro.....94/ Itm. media fanega de Sal vte. Y cuatro/ Itm. ochenta a(rrobas) de Carbon trescientos quarta. ......340/ Itm. una a(rroba) de Arroz treinta y dos.....32/ Itm.. otra Id de Judias Veinte y dos....22/ Itm. dos Id de vino ochenta y ocho.....88./ Itm. en dinero efectivo seis mil Rs.on.......6.000/ 285.407.../ Importan los bienes expresados ante/ riormente la cantidad de doscientos ochenta y cinco mil/ quatrocientos siete rs. Salvo error, a saber los doscien/tos Seis mil quinientos Veinte y Cinco, por dona/cion hecha al D. Pio por su Abuelo D. Francisco/ (fol 32)

de Goya, y los Setenta y ocho mil ochocientos/ ochenta y dos, que le han dado, y han salido/ del cuerpo de bienes de sus Padres por cuenta de sus / legitimas paterna y materna, de que los otorgantes/ se dan por contentos y satisfechos a toda su voluntad/ por servirlos en este acto del D. Pio Mariano de Goya, y por mano de sus Padres D Francisco Xavier y D $D^{a}$ Gumersinda Goicoechea, y en cuanto a las/ raices las Escrituras de su pertenencia a mi presen/ cia y de los testigos, que se expresaran de que doy/fee, y como real y efectivamente satisfecho de/ ellos formaliza a su favor el resguardo mas fir/ me y eficaz, que a su seguridad conduzca, y de/ claran que los bienes referidos han sido valuados/ por personas inteligentes electas de conformidad de/ los citados D. Francisco Xavier Pedro de Goya y /de D. Francisco Xavier de Mariategui, Padres de/ los Novios, y que en su tasacion no ha havido/ lesion ni engaño, y por lo mismo la apruevan y / ratifican, y se obligan a no reclamarla y si lo hicieran sea visto por lo mismo haverla aprovado nue/vamente añadiendo fuerza a fuer$\mathrm{za}$, y contrato/ a Contrato. Y en su consecuencia se obliga la/ $\mathrm{D}^{\mathrm{a}}$ Maria Valvanera de la Concepcion de Mareate/ gui a tomar por caudal del citado su futuro eEs/poso todos los mencionados bienes, y los que han de / (fol. 32 vto.)y adquirir por donacion, u otro Contrato de algun Pariente/ u extraño para que no le perjudique cuando el matrimo./ se disuelva a lo que quiera se les consuele en legal forma./ Y al cumpto. De lo referido 
obliga sus bienes presentes, y futuros, por amplio poder a los Sres. Jueces de esta Villa para/ que la Compelan como por Sentencia definitiva pa-/ sada en autoridad de cosa juzgada y consentida, que / por tal la recive, renuncia las Leyes fueros, y privi/ legios de su favor, memoria de edad, y bene ficio de / restitucion ni integracion que le compete. Y asi lo / otorgan, y firman, a quienes doy fee conozco siendo / testigos D. Bernardino Tolosa, D. Juan Jose Sanchez / Pescador y D Jose Salanova, vecinos y residentes en esta Corte./

Maria de la Concepcion Mariategui, (rubricado) Franc ${ }^{a}$ Javier de Mariategui (rubricado) / Ramona Asperilla (rubricado) Antemy/ Antonio Lopez de Salazar (rubricado)».

JuAN JoSÉ JunQuera y MATO

\section{ADDENDA AL CAPRICHO DE GOYA «TÚ QUE NO PUEDES»}

En el número de Archivo Español de Arte, correspondiente a diciembre del 2004, puse en relación formal - no de contenido- algunos grabados de los Caprichos, con otros que ilustraban un Exemplario, publicado en Zaragoza en 1531, y que pudieron servir de inspiración al pintor aragonés ${ }^{1}$.

Revisando el libro de Randall, sobre márgenes miniados, he tropezado con un motivo más cercano al capricho Tú que no puedes (fig. 1), que el propuesto en mi anterior trabajo. Se trata de una miniatura en la que aparece un hombre cargando un asno sobre sus espaldas (fig. 2), de la misma manera que en la escena goyesca. Randall sugiere que puede tratarse de la ilustración de un proverbio o de una fábula ${ }^{2}$.

Relacionado con esta escena existe un refrán recogido por el Marqués de Santillana en su Refranero: «No es de seso traer el asno en peso», y que Antonio Castillo de Lucas identifica con la insensatez del hombre ${ }^{3}$, interpretación que puede concordar con lo expresado por Goya en su grabado.

También, releyendo la Nave de los Locos de Sebastián Brandt, publicada en Basilea en 1493, vemos una escena similar (fig. 3), con un epígrafe referido a la estupidez humana, especialmente representada por los insensatos que se dejan abrumar por cualquier motivo, y que también podría convenir como precedente literario y artístico del grabado de Goya: «Muchos necios que estultos son en múltiples sentidos, están en este aprieto: sentadoles está el asno en las espaldas» 4 .

No se ha sabido nunca si Goya tuvo una biblioteca, pero tanto el Exemplario, como el Refranero de Santillana y la Nave de los locos, podrían haber sido libros que, por su temática, no solo era lógico de contar en la biblioteca de Iriarte, por ejemplo, sino como propiedad del mismo Goya, ya que el contexto de ellas casaba perfectamente con el talante sentencioso y popular de algunas producciones del pintor aragonés.

IsABel MATeo GómEZ

${ }^{1}$ I. Mateo Gómez, «A propósito de algunos Caprichos de Goya y un Exemplario aragonés de 1531», Archivo Español de Arte, LXXVII, 2004, pp. 411-415, figs. 1 y 2. Involuntariamente omití en este trabajo, que el Exemplario me fue regalado por Benito Navarrete, a quien agradezco, además, no pocas opiniones.

${ }^{2}$ L. M. C. Randall, Images in the margins of Gothic manuscripts, Berkeley, 1966, p. 331.

${ }^{3}$ A. Castillo de Lucas, «Ensayo antropológico del Marqués de Santillana y de su Refranero», Archivo Iberoamericano de Historia de la medicina y Antropología médica, vol. XI, fasc. 3, 1959, pág. 37, nº 490 (en el Marqués de Santillana, refrán $n^{\circ} 477$ )

${ }^{4}$ Ed. Akal, 1998, pp. 244-245.

$A E A$, LXXVIII, 2005, 309, pp. 83 a 105 